\title{
ON THE CROSSING OF INTERMEDIATE UNSTABLE STEADY STATE SOLUTIONS FOR THERMAL IGNITION IN A SPHERE
}

\author{
R. O. WEBER ${ }^{1}$, G. C. WAKE ${ }^{2}$, H. S. SIDHU ${ }^{1}$, G. N. MERCER ${ }^{1}$, B. F. GRAY ${ }^{3}$, \\ W. DERRICK ${ }^{4}$ and E. BALAKRISHNAN ${ }^{5}$
}

(Received 29 November, 1998; revised 25 March, 1999)

\begin{abstract}
Steady state solutions for spontaneous thermal ignition in a unit sphere are considered. The multiplicity of unstable, intermediate, steady state, temperature profiles is calculated and shown for selected parameter values. The crossing of the temperature profiles corresponding to the unstable, intermediate, steady states is exhibited in a particular case and is proven in general using elementary ideas from analysis. Estimates of the location of crossing points are given.
\end{abstract}

\section{Introduction}

Models of spontaneous thermal ignition have a long history, beginning with the Russian literature as listed by Frank-Kamenetskii [7]. A parallel literature in astrophysics has examined many similar problems; for example, Chandrasekhar [5]. In both cases, spherically geometric temperature distributions have been of considerable interest and numerical, as well as analytical, effort has been expended in the cause of obtaining complete solutions.

It was first recognised by Gel'fand [9], Fujita [8] and Steggerda [18] (see also Enig [6], Gray [11], Aris [1, p. 48] and Zeldovich et al. [20, p. 159]) that there can be a very large number of solutions for the steady-state, reaction-diffusion equation in spherical geometry with an exponential reaction term or a polynomial reaction term of sufficiently high degree (greater than 5). This was initially regarded as a

\footnotetext{
ISchool of Mathematics and Statistics, University College, University of New South Wales; Australian Defence Force Academy, Canberra 2600, Australia.

${ }^{2}$ Department of Mathematics, University of Canterbury, Christchurch, New Zealand.

${ }^{3}$ School of Chemistry, Macquarie University, Sydney, NSW, Australia.

${ }^{4}$ Department of Mathematics, University of Montana, Missoula, MT, USA.

${ }^{5}$ Department of Mathematics and Statistics, Sultan Qaboos University, Sultanate of Oman.

(C) Australian Mathematical Society 2001, Serial-fee code 0334-2700/01
} 
mere mathematical curiosity, but later investigators have wondered if there could be a physical role for the gross multiplicity of steady states, as well as examining the bifurcation in greater detail; for example Ash et al. [2], Brindley et al. [4], Balakrishnan et al. [3], Kapila et al. [14], Maddocks [15], Weber et al. [19].

In the present paper, we wish to examine the spatial distributions of these multiple steady states. We will demonstrate numerically and then prove rigorously that the existence of multiple unsteady steady states necessitates that their spatial distributions must cross (already suggested diagrammatically, but never formally stated in earlier work as reported by Zeldovich et al. [20, p. 160]). Furthermore, we will give estimates for the crossing points and the associated function values.

\section{Steady state solutions for spontaneous thermal ignition in a sphere}

If we seek a balance between the effect of diffusional fluxes of heat and an exothermic reaction $f(u)$, then it can be shown (Frank-Kamenetskii [7], Weber, Wake and Balakrishnan [19] and references therein) that the temperature variable $u$ must satisfy

$$
\nabla^{2} u+f(u)=0 .
$$

This equation represents a steady-state and non-dimensionalised statement of the principle of conservation of energy and as such is the starting point for many investigations into thermal ignition theory.

In earlier modelling and theoretical work, the reaction function $f(u)$ was taken as being proportional to the exponential function (that is, $\delta e^{u}$ ). However, more recently, it has been conclusively demonstrated by Gray and Wake [12] that there are tangible benefits (such as a more simple correspondence between the ambient temperature and bifurcation parameters) if the full temperature dependence of the Arrhenius function is retained. Consequently, we shall focus our investigation on (1) with $f(u)=\lambda e^{-1 / u}$ ( $\lambda$ is a constant dependent upon the thermo-physical properties of the exothermic material under consideration). Furthermore, Gidas, Ni and Nirenberg [10] have shown that all positive solutions of (1) satisfying Dirichlet boundary conditions are radially symmetric, so we use spherical coordinates and (1) then becomes

$$
\frac{d^{2} u}{d r^{2}}+\frac{2}{r} \frac{d u}{d r}+\lambda e^{-1 / u}=0 .
$$

Naturally this equation will need to be solved for the temperature $u$, as a function of the radial coordinate $r$ and the parameter $\lambda$, subject to the appropriate Dirichlet boundary conditions.

In order to keep the subsequent analysis and presentation of results reasonably simple, we shall restrict our study to the case where the exterior is held at a constant 


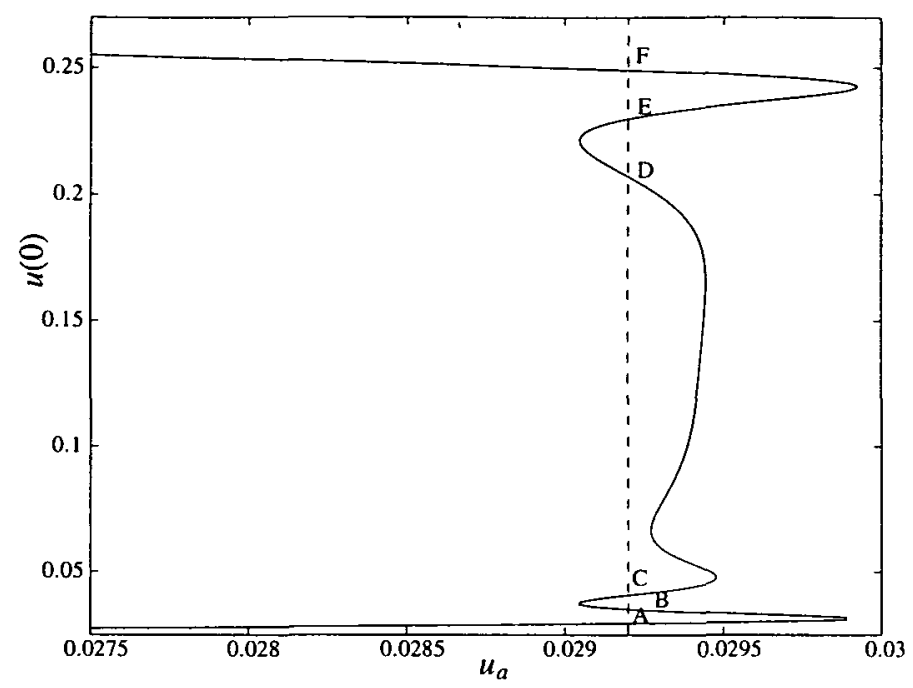

FIGURE 1. Bifurcation diagram showing the central temperature $u(0)$ as a function of ambient temperature $u_{a}$ for fixed $\lambda=10^{12}$ for a unit sphere. Unstable, intermediate, steady state profiles are labelled B, C, D, $\mathrm{E}$ and $\mathrm{F}$ for $u_{a}=0.0292$.

temperature; called the ambient temperature and denoted $u_{a}$. Due to the use of dimensionless parameters, we can assume, without loss of generality, that the sphere has unit radius. Hence our boundary conditions can be written as

$$
\begin{aligned}
\left.\frac{d u}{d r}\right|_{r=0} & =0, \\
u(1) & =u_{a} .
\end{aligned}
$$

The first of these is merely a statement requiring bounded solutions at the centre of our spherically symmetric domain. Additionally, it should be noted that our choice of reasonably simple boundary conditions is not essential for the surprisingly complex solutions which we will display. It does, however, make our task of presenting, explaining and analysing the results somewhat easier and (hopefully) less confusing.

Solutions of (2) subject to boundary conditions (3) need to be constructed numerically (with the possible exception of a series approach for the simple exponential reaction as detailed in Frank-Kamenetskii [7]). Certainly there has been considerable effort in this direction and a consistent picture of the results has emerged. For sufficiently large $\lambda$ and for some $0<u_{a}<1 / 4$, there is found to exist one low temperature solution, stable to temporal perturbations, and one high temperature solution, also stable to temporal perturbations. In addition, there exists a large number of intermediate solutions, all of which are unstable to temporal perturbations. This 

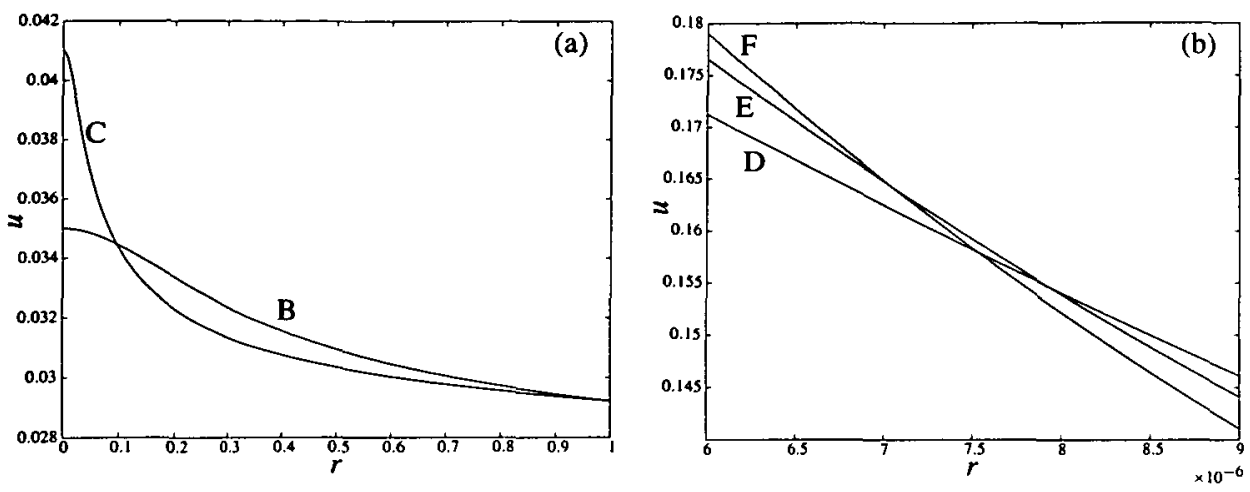

FIGURE 2. Unstable, intermediate, steady state profiles corresponding to points in Figure 1 (that is, $u_{a}=0.0292$ ). Note the different scales in (a) and (b) required in order to clearly display these profiles.

is best displayed and understood with a bifurcation diagram, plotting the maximal steady state temperature $u(0)$ as a function of a bifurcation parameter; for example $u_{a}$, and holding the other parameter, $\lambda$, constant. In Figure 1 , the result for $\lambda=10^{12}$, a realistic value for milk powder (see Weber $e$ t al. [19]), is shown and it can be clearly seen that for certain values of $u_{a}$, there exist five intermediate steady states; all of which turn out to be unstable if analysed by a linear, temporal, stability analysis or if analysed numerically. Note that due to scaling difficulties, the high temperature branch is not shown on Figure 1.

The bifurcation diagram was obtained using MATLAB ${ }^{\mathrm{TM}}$. The results were checked by using the path following software AUTO97.

\section{Spatial profiles of steady state solutions}

Spatial profiles of the steady state temperature were also obtained numerically by a shooting method; integrating from the already known central temperature out to the temperature at the exterior of the sphere $\left(u_{a}\right)$.

As an example we display in Figures 2 (a) and 2 (b) the results for the points labelled B, C, D, E and F in Figure 1 . It is apparent that these five points all have the same values for the parameters $\lambda$ and $u_{a}$, yet they correspond to different, unstable, intermediate, temperature profiles. Furthermore, it can be clearly seen that these profiles cross each other so that the one with the highest value for $u(0)$ ends up below all of the others near the exterior of the sphere. To see this, it is necessary to note that different scales were required on the axes so that the distributions could be clearly displayed. We also note the natural ordering of the crossing points, as indicated schematically in Figure 3. Namely, if $u_{i}(0)<u_{j}(0)<u_{k}(0)$ for intermediate steady states, then the coordinate values where crossing occurs is given by $r_{j k}<r_{i k}<r_{i j}$. Part of this will be rigorously 


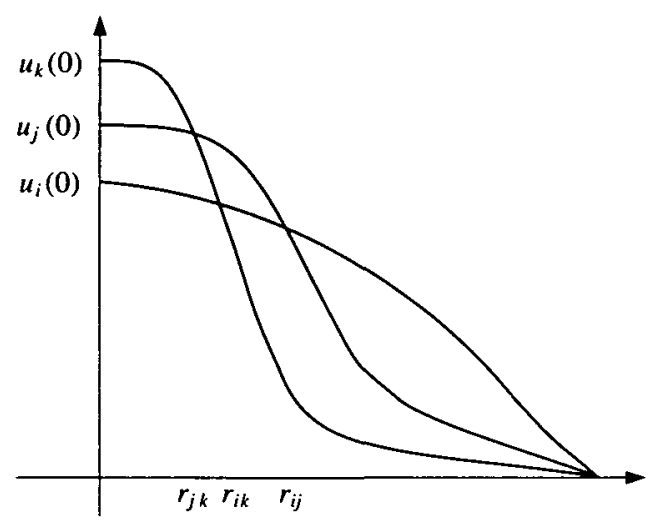

FIGURE 3. Schematic of the unstable, intermediate, steady state profiles showing the ordering of the crossing points.

proven in Section 5.

\section{Crossing as a natural requirement of the evolution of initial conditions}

Having noted from the numerical results that the spatial distributions of the unstable intermediate steady states cross, we naturally wonder if this is a general requirement, or if we are considering a special case. Prior to proving that this is indeed a general requirement (in the next section) we shall consider reasons for this which follow from ideas on the evolution of initial conditions applied to the semi-linear parabolic equation

$$
u_{t}=u_{r r}+\frac{2}{r} u_{r}+\lambda e^{-1 / u}
$$

Assume that there exist two, unstable, intermediate, steady state profiles as solutions to (4) with boundary conditions (3), that their profiles do not cross on $r \in[0,1]$ and that there is no stable steady state profile in between these two. Then, as an initial condition begin with the average of these two, unstable, intermediate, steady state profiles. Clearly the evolution of this initial profile will be constrained to lie between the unstable profiles (for such equations, extremum principles, for example, Protter and Weinberger [17], prove that profiles everywhere below cannot cross at some later time). At the same time, the initial profile cannot find a steady state to evolve towards, so it would seem that it must be ever changing in time. Norbury and Wake [16] have proven that evolving solutions to partial differential equations such as (4) cannot oscillate with a fixed frequency. Consequently the only fate for such an initial profile 
would be to evolve randomly in time while being constrained to lie in between the two unstable, intermediate, steady state profiles. This is a most unlikely scenario and we are led to conclude that our supposition that the profiles do not cross is incorrect. Hence we expect the crossing of unstable, intermediate, steady state profiles to be required.

\section{Crossing proof and estimates for crossing points}

Multiply (2) by $r^{2}$ and integrate from 0 to $r$, obtaining

$$
r^{2} u^{\prime}(r)=-\int_{0}^{r} s^{2} f(u(s)) d s<0,
$$

so that every solution $u(r)$ of (2) and (3) is monotone decreasing on [0,1]. Define $g\left(u, u_{i}\right)=\left(f(u)-f\left(u_{i}\right)\right) /\left(u-u_{i}\right)$ (where $u_{i}$ is some solution to be defined later) and differentiate with respect to $u$ yielding

$$
\frac{\partial g}{\partial u}=\frac{f(u)}{u^{2}\left(u-u_{i}\right)^{2}}\left[u-u_{i}+u^{2}\left(\frac{f\left(u_{i}\right)}{f(u)}-1\right)\right]=\frac{f(u)}{u^{2}\left(u-u_{i}\right)^{2}} G(u)
$$

with $G(u)$ defined to be the contents of the square bracket. Then $G\left(u_{i}\right)=0$ and $G^{\prime}(u)=(2 u-1)\left(f\left(u_{i}\right) / f(u)-1\right)>0$, for $u_{i}<u<0.5$, so $G(u)$ is positive and $g\left(u, u_{i}\right)$ is strictly increasing in $u_{i}<u<0.5$. Denote the low temperature stable solution by $u_{\min }(r)$, then any other solution $u(r)$ must satisfy $u_{\min }(r) \leq u(r)$ at every point in the domain $0 \leq r \leq 1$.

THEOREM 1. Let $u_{1}(r)$ and $u_{2}(r)$ be two intermediate solutions of (2) and (3), with $u_{\min }(0)<u_{1}(0)<u_{2}(0)<0.5$. Then there is at least one value $0<r_{12}<1$ such that $u_{1}\left(r_{12}\right)=u_{2}\left(r_{12}\right)$.

PRoOF. Assume that $u_{2}>u_{1}$ on $[0,1)$ and define $v_{i}=u_{i}-u_{\min }, i=1,2$, so that $v_{2}>v_{1}>0$ in $[0,1)$ and $v_{1}(1)=v_{2}(1)=0$. By Green's theorem

$$
\int_{|r|<1}\left(v_{1} \nabla^{2} v_{2}-v_{2} \nabla^{2} v_{1}\right) d V=\int_{|r|=1}\left(v_{1}\left(v_{2}\right)_{n}-v_{2}\left(v_{1}\right)_{n}\right) d S=0 .
$$

However, $\nabla^{2} v_{i}=\nabla^{2} u_{i}-\nabla^{2} u_{\min }=f\left(u_{\min }\right)-f\left(u_{i}\right)$, so that (7) becomes

$$
0=\int_{|r|<1}\left(v_{1} v_{2}\left[g\left(u_{1}, u_{\min }\right)-g\left(u_{2}, u_{\min }\right)\right]\right) d V<0,
$$

which is a contradiction, since $g\left(u_{1}(r), u_{\min }(r)\right)<g\left(u_{2}(r), u_{\min }(r)\right)$ as $g$ is strictly increasing. 
THEOREM 2. Let $u_{1}, u_{2}$, and $u_{3}$ be three intermediate solutions of (2) and (3), with $u_{\min }(0)<u_{1}(0)<u_{2}(0)<u_{3}(0)<0.5$. Then $r_{23}<r_{13}$ and $r_{23}<r_{12}$.

Proof. The functions $z=u_{3}-u_{1}$ and $w=u_{2}-u_{1}$ satisfy the boundary value problems

$$
\begin{aligned}
\left(r^{2} z^{\prime}\right)^{\prime}+r^{2} g\left(u_{1}, u_{3}\right) z & =0, & z^{\prime}(0) & =z(1)=0, \\
\left(r^{2} w^{\prime}\right)^{\prime}+r^{2} g\left(u_{1}, u_{2}\right) w & =0, & w^{\prime}(0) & =w(1)=0 .
\end{aligned}
$$

Assume $r_{23} \geq r_{12}$, so that $u_{3}>u_{2}>u_{1}$ and $g\left(u_{1}, u_{2}\right)>g\left(u_{1}, u_{2}\right)$ on $|r|<r_{12}$. Multiply the first equation in (9) by $w$ and the second by $z$ and subtract:

$$
\left(r^{2} w^{\prime}\right)^{\prime}-\left(r^{2} w^{\prime}\right)^{\prime} z+r^{2}\left[g\left(u_{1}, u_{3}\right)-g\left(u_{1}, u_{2}\right)\right] z w=0 .
$$

Integrating over $\left(0, r_{12}\right)$ yields

$$
\begin{aligned}
0 & <\int_{0}^{r_{12}} r^{2}\left[g\left(u_{1}, u_{3}\right)-g\left(u_{1}, u_{2}\right)\right] z w d r=-\int_{0}^{r_{12}}\left[\left(r^{2} z^{\prime}\right)^{\prime} w-\left(r^{2} w^{\prime}\right)^{\prime} z\right] d r \\
& =\left[\left(r^{2} w^{\prime}\right) z-\left(r^{2} z^{\prime}\right) w\right]_{0}^{r_{12}}=r_{12}^{2} z\left(r_{12}\right) w^{\prime}\left(r_{12}\right)<0
\end{aligned}
$$

which is impossible. Hence $r_{23}<r_{12}$ and by continuity it follows that $r_{23}<r_{13}$.

We have not been able to prove that $r_{13}<r_{12}$ although the numerical profiles suggest this is true. The proofs of Theorems 1 and 2 can be generalized to $n$-dimensional space.

THEOREM 3. Let $u_{1}$ and $u_{2}$ be two intermediate solutions of (2) and (3), with $u_{\min }(0)<u_{1}(0)<u_{2}(0)<0.5$. Then, if $\rho$ is the last value in $(0,1)$ at which $u_{1}(\rho)=u_{2}(\rho)$,

$$
4<\frac{4}{(1-\rho)^{2}} \leq f^{\prime}\left(u_{1}(\rho)\right)<f^{\prime}\left(u_{1}(0)\right)
$$

Proof. Let $w=u_{2}-u_{1}$ satisfy (9). Then $y=r w$ is a nontrivial solution of the boundary value problem in region $(\rho, 1)$ :

$$
y^{\prime \prime}+g\left(u_{1}, u_{2}\right) y=0 \quad \text { in }(\rho, 1), y(\rho)=y(1)=0 .
$$

By Lyapunov's theorem (see for example Hartman [13, p. 346]), since $y$ has at least two zeros in $[\rho, 1]$,

$$
\frac{4}{1-\rho}<\int_{\rho}^{1} g\left(u_{1}(r), u_{2}(r)\right) d r \leq g\left(u_{2}(\rho), u_{2}(\rho)\right)(1-\rho)=f^{\prime}\left(u_{1}(\rho)\right)(1-\rho) .
$$


REMARK. Since $f^{\prime}(u)$ is monotone increasing in $0<u<0.5$, it is useful to determine where $f^{\prime}\left(u_{0}\right)=4$. For example this occurs when $\lambda=10^{12}$ at approximately $u_{0}=0.0300724586$. Consequently all pairs of intermediate solutions must have their last crossover above $u_{0}$. This is certainly the case in Figure 2. Further, the initial values of the intermediate solutions must exceed $u_{0}$, suggesting that $u_{0}$ determines the rightmost limit point in Figure 1.

\section{Conclusion}

The evolution of certain initial conditions is clearly influenced by the crossing of the infinity of unstable steady states. We have proven and demonstrated interesting properties of the spatially distributed unstable steady states, in particular the crossing over of the distributions which is a necessary consequence of well known properties of the ordinary differential equation, as well as the instability of the intermediate steady states. The consequences for the crossing with the inclusion of fuel consumption is currently under investigation.

\section{Acknowledgements}

ROW gratefully acknowledges the hospitality of the University of Auckland and the generosity of the Marsden fund for the initial phase of this work carried out while ROW was on sabbatical from the UNSW. WD gratefully acknowledges the hospitality and support of UQ and UNSW for work done on this topic while WD was on sabbatical from the University of Montana. The authors would also like to acknowledge the advice of John Billingham for the numerical computaion of the bifurcation diagram.

\section{References}

[1] R. Aris, The mathematical theory of diffusion and reaction in permeable catalysts, Vol. II (Clarendon Press, Oxford, 1975).

[2] E. Ash, B. Eaton and K. Gustafson, "Counting the number of solutions in combustion and reactive flow problems", J. Appl. Math. Phys. (ZAMP) 41 (1990) 558-578.

[3] E. Balakrishnan, A. Swift and G. C. Wake, "Multiple solutions in hollow geometries in the theory of thermal ignition", Appl. Math. Lett. 10 (5) (1997) 41-46.

[4] J. Brindley, N. A. Jivraj, J. H. Merkin and S. K. Scott, "Stationary-state solutions for coupled reaction-diffusion and temperature-conduction equations II. Spherical geometry with Dirichlet boundary conditions", Proc. R. Soc. Lond. A 430 (1990) 479-488.

[5] S. Chandrasekhar, An introduction to the study of stellar structure (Dover, New York, 1967) Ch. IV, 84-182. 
[6] J. W. Enig, "Critical parameters in the Poisson-Boltzmann equation of steady state thermal explosion theory", Combust. Flame 10 (1966) 197-199.

[7] D. A. Frank-Kamenetskii, Diffusion and heat transfer in chemical kinetics, 2nd ed. (Plenum Press, New York, 1969).

[8] H. Fujita, "On the nonlinear equation $\delta u+e^{u}=0$ and $v_{t}=\delta v+e^{v ", ~ B u l l . ~ A m e r . ~ M a t h . ~ S o c . ~} 75$ (1965) 132-135.

[9] I. M. Gel' fand, "Some problems in the theory of quasilinear equations", Amer. Math. Soc. Trans. Series 229 (1963) 295-381.

[10] B. Gidas, W. N. Ni and L. Nirenberg, "Symmetry and related properties via the maximum principle", Comm. Math. Phys. 68 (1979) 209-243.

[11] B. F. Gray, "On the critical conditions in a spherical reacting mass", Combust. Flame 73 (1969) 50-54.

[12] B. F. Gray and G. C. Wake, "Critical initial conditions for thermal ignition", Math. Comput. Modelling 18 (8) (1993) 65-75.

[13] P. Hartman, Ordinary differential equations (Wiley, New York, 1964).

[14] A. K. Kapila, B. J. Matkowsky and J. Vega, "Reactive-diffusive system with Arthenius kinetics: peculiarities of the spherical geometry", SIAM J. Appl. Math. 38 (1980) 382-401.

[15] J. H. Maddocks, "Stability and folds", Archive Rat. Mech. Anal. 99 (1987) 301-328.

[16] J. Norbury and G. C. Wake, "Spatial pattern formation for steady states of a population model", IMA J. Math. Appl. in Medicine \& Biology 10 (1993) 19-30.

[17] M. H. Protter and H. F. Weinberger, Maximum principles in differential equations (Springer, New York, 1984).

[18] J. J. Steggerda, "Thermal stability: an extension of Frank-Kamenetskii's theory", J. Chem. Phys. 43 (1965) 4446-4448.

[19] R. O. Weber, G. C. Wake and E. Balakrishnan, "Critical initial conditions for spontaneous thermal ignition", Faraday Trans. 94 (1998) 3613-3617.

[20] Ya. B. Zeldovich, G. I. Barenblatt, V. B. Librovich and G. M. Makhviladze, The mathematical theory of combustion and explosions (Consultants Bureau, Plenum, New York, 1985). 